\title{
P02-1-72 Poster session
}

\section{Downregulation of astrocytic connexin43 leads to enhancement of neurotrophic factor production in cortical astrocytes}

\author{
Syun Kondo, Kazue Hisaoka-Nakashima, Yoshihiro Nakata, Norimitsu Morioka \\ Department of Pharmacology, Hiroshima University Graduate School of Biomedical \& Health Sciences, Japan
}

background:

Connexin43 ( $\left.\mathrm{Cx}_{\mathrm{X}} 43\right)$, involved in intercellular signaling, is expressed in astrocytes. Some studies show that downregulation of $\mathrm{C}_{x} 43$ induces depressive-like behaviors in rats. Brain-derived neurotrophic factor (BDNF) and glial cell line-derived neurotrophic factor (GDNF) produced from cortical astrocytes could be crucial players in pathology of depression. In this study, we investigated whether astrocytic $\mathrm{Cx} 43$ might be involved in regulation of neurotrophic factors expression.

methods:

Primary cultured astrocytes were prepared from the cortex of neonatal Wister rats. Cx43 knockdown in cultured astrocytes was performed using RNA interference.

results:

We first examined the effect of $\mathrm{Cx} 43$ knockdown on noradrenaline (NA)-induced BDNF mRNA and GDNF mRNA expression. As a result, significant enhancement of NA-induced BDNF and GDNF mRNA expression was observed in astrocytes transfected with $\mathrm{Cx} 43$ siRNA compared to those with non-targeting siRNA. These responses were inhibited by treatment with U0126, which is an inhibitor of extracellular signal-regulated kinase (ERK). Furthermore, NA-induced ERK phosphorylation was potentiated in $\mathrm{Cx} 43$ siRNA-transfected astrocytes. In addition, NA-induced phosphorylation of cAMP response element binding protein $(\mathrm{CREB})$, which is a transcriptional regulatory factor mediating expression of both BDNF and GDNF, was also enhanced in Cx43 siRNA-transfected astrocytes.

conclusions:

In the current study, downregulation of $\mathrm{Cx}_{\mathrm{x}} 43$ could potentiate NA-induced BDNF mRNA and GDNF mRNA expression. Enhancement of ERK-CREB activity is involved in Cx43 knockdown-induced neurotrophic factor expression. These results suggest that astrocytic $\mathrm{C}_{\mathrm{X}} 43$, which is downregulated in depression, is involved in regulation of neurotrophic factors expression, and downregulation of astrocytic $\mathrm{Cx} 43$ might affect functioning and reactivity of astrocytes in depression. 